\title{
ON THE SENSITIVITY OF THE BEHAVIOUR OF STELLAR ORBITS TO THE PARAMETERS OF SIMPLE GALACTIC MASS MODELS
}

\author{
LOUIS MARTINET
}

Observatoire de Genève, Sauverny, Switzerland

\begin{abstract}
Some conjectures are given about the sensitivity of the behaviour of the stellar orbits (appearance of resonant orbits, possible interaction of resonances etc.) to the set of parameters characterizing a simple realistic 'spheroid + nucleus' mass model of our Galaxy. Connections with a systematic exploration of orbits in Schmidt mass models are mentioned. Finally we present arguments in favour of the existence of a third integral for the whole solar neighbourhood old stellar populations, independently of the chosen model.
\end{abstract}

\section{Introduction}

In recent years, we have extensively studied the orbital properties of old stellar populations in our Galaxy represented by an axisymmetric stationary potential $\Phi(\varpi, z)$ (Martinet and Hayli, 1972 (I); Mayer and Martinet, 1973 (II); Martinet and Mayer, 1975 (III)). In fact these objects can give us information on the early stages of the dynamic galactic evolution. But the point is: What are the changes in the orbits when the proposed force function changes so that the rotation curve or the acceleration perpendicular to the galactic plane, as well as the constants used in the model, take all permissible values within the limits set by the errors of observation? For our systematic exploration of galactic orbits, we used both versions of a galactic model given by Schmidt in 1956 and 1965 (noted I and II henceforth). We shall not discuss here what should be the 'best' mass model of our Galaxy. Until recently, the Schmidt models were those which permitted the computation of numerous orbits without excessive expense of time. The main types of features we have found in our exploration also appear in Innanen's models which seem to answer better the criteria of the 'best model' (Innanen et al., 1972). It is not easy to answer the above question by comparing the results from both versions of the Schmidt potential, because for the first we use an interpolation formula and the 2 nd includes a nucleus, a very flattened spheroid and an outer shell. Here we shall use some results which we have obtained in the three papers mentioned before as a guide and as a checking for a first approach of a more general study which consists of seeing the influence of different accessible parameters which characterize a galactic mass model on the existence of important resonances and on the possible appearance of 'wild' behaviour of orbits. Having a care for using the 'best' model, we ought to include in a complete theory a superposition of non homogeneous spheroids but the calculations which follow seem sufficient in order to understand the main effects, at least for a first approach. 


\section{Oscillations in the Meridian Plane}

Let us consider the classical expansion for the density law corresponding to the potential $\Phi(\varpi, z)$ for a flattened spheroid,

$$
\varrho(\alpha)=\frac{p_{-2}}{\alpha^{2}}+\frac{p_{-1}}{\alpha}+p_{0}+p_{1} \alpha+p_{2} \alpha^{2}+\cdots \quad \text { with } \quad \alpha=\sqrt{\varpi^{2}+\left(1-e^{2}\right)^{-1} z^{2}}
$$

to which corresponds, for the rotation velocity $\Theta_{c}(\varpi)$, the expansion

$$
\frac{\Theta_{c}^{2}}{\varpi^{2}}=\frac{v_{-2}}{\varpi^{2}}+\frac{v_{-1}}{\varpi}+v_{0}+v_{1} \varpi+v_{2} \varpi+\cdots
$$

by using the relation

$$
\Theta_{c}^{2}=4 \pi G \sqrt{1-e^{2}} \int_{0}^{w} \frac{\varrho(\alpha) \alpha^{2} \mathrm{~d} \alpha}{\sqrt{\varpi^{2}-\alpha^{2} e^{2}}}
$$

with $e=$ eccentricity of the spheroid.

It is easy to compute the ratio $\left(A_{c} / B_{c}\right)^{1 / 2}$ of the infinitesimal oscillation frequencies in a meridian orbital plane, where the motion takes place, controlled by the 'reduced' potential

$$
U(\varpi, z)=\frac{J^{2}}{\varpi^{2}}+\Phi(\varpi, z)
$$

$J$ being the angular momentum.

Expanding $U$ around $\varpi=\varpi_{c}, z=0$, where $\varpi_{c}$ is defined by

we have

$$
\frac{J^{2}}{w_{c}^{3}}=\left(\frac{\partial \Phi}{\partial \varpi}\right)_{w=w_{c}}
$$

where

$$
U(\varpi, z)=U_{0}+\frac{1}{2}\left(A_{c}\left(\varpi-\varpi_{c}\right)^{2}+B_{c} z^{2}\right)-\varepsilon\left(\varpi-\varpi_{c}\right) z^{2}-\eta\left(\varpi-\varpi_{c}\right)^{3}+\cdots,
$$

$$
A_{c}=\frac{3 J^{2}}{\varpi_{c}^{3}}+\left(\frac{\partial \Phi}{\partial \varpi}\right)_{\substack{w=w_{c} \\ z=0}}=-\left(\frac{3 K_{w}}{\varpi}+\frac{\partial K_{w}}{\partial \varpi}\right)_{\substack{w=w_{c} \\ z=0}}
$$

and

$$
B_{c}=-\left(\frac{\partial^{2} \Phi}{\partial z^{2}}\right)_{\substack{w=\Phi_{c} \\ z=0}}=\left(\frac{K_{w}}{\varpi}+\frac{\partial K_{w}}{\partial \varpi}\right)_{\substack{w=\Phi_{c} \\ z=0}}+4 \pi G \varrho\left(\varpi_{c}\right) .
$$

With the previous relations and with

$$
J_{n}=\frac{e^{n}}{\sqrt{1-e^{2}}} / \int_{0}^{\infty} \sin ^{n-1} \vartheta \mathrm{d} \vartheta
$$




$$
\frac{A_{c}}{B_{c}}=\frac{\frac{2 v_{-2}}{\varpi_{c}^{2}}+\frac{3 v_{-1}}{\varpi_{c}}+4 v_{0}+5 v_{1} \varpi_{c}+6 v_{2} \varpi_{c}^{2}+\frac{C}{\varpi_{c}^{3}}}{\frac{J_{1} v_{-2}}{\varpi_{c}^{2}}+\frac{\left(J_{2}-1\right) v_{1}}{\varpi_{c}}+\left(J_{3}-2\right) v_{0}+\left(J_{4}-3\right) v_{1} \varpi_{c}+\left(J_{5}-4\right) v_{2} \varpi_{c}^{2}+\frac{C}{\varpi_{c}^{3}}},
$$

where the term $C / \varpi_{c}^{3}$ has been introduced in order to take into account the possible existence of a massive nucleus. Two features must be emphasized: (a) Without the presence of a nucleus or in regions where the influence of such a nucleus is not important, the ratio $\left(A_{c} / B_{c}\right)^{1 / 2}$ is approximately constant on a large range of $\varpi_{c}$, as long as $e$ is not too far from 1 (thus this property is connected with the mode of construction of the models as well as with the eccentricity of the spheroids); (b) The presence of a massive nucleus introduces a significant variation of $\left(A_{c} / B_{c}\right)^{1 / 2}$ essentially for small $\varpi_{c}$ These features appear in Figure 1 which shows the influence of $e$ and of the mass of the nucleus on the ratio $\left(A_{c} / B_{c}\right)^{1 / 2}$ for a model in which only $v_{-1}$ and $v_{1}$ are different from zero. As shown below, $\left(A_{c} / B_{c}\right)^{1 / 2}$ is also modified if we change the value of the mass density at the Sun, $\varrho_{\odot}$ (which is used by Schmidt to fix the value of $e$ ). It is easy to repeat the discussion for a density law corresponding to another choice of the coefficients $v_{i} \neq 0$ (i.e. $v_{0}$ and $v_{-1}$ ). The main features mentioned below remain unchanged.

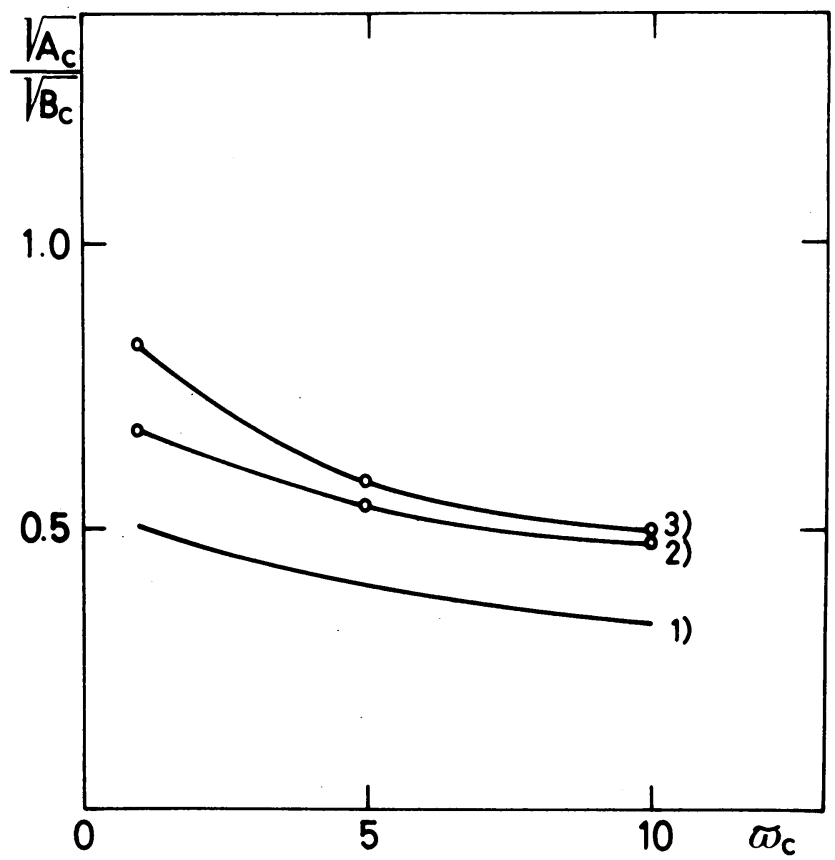

Fig. 1. Ratio of oscillation frequencies in the meridian plane $\left(A_{c} / B_{c}\right)^{1 / 2}$ vs $\varpi=\varpi_{c}$ for a 'nucleus + spheroid' model characterized by a rotation curve $\Theta_{c}=\sqrt{v_{-1} \varpi+v_{1} \varpi^{3}+(C / \varpi)}$ for different values of the parameters: (a) $v_{-1}=10000, v_{1}=-40, C=30000$ and $e=$ eccentricity of the spheroid $=0.9988 ;$ (b) $v_{-1}=10000$, $v_{1}=-40, C=30000$ and $e=0.995 ;$ (c) $v_{-1}=10000 ; v_{1}=-40, C=120000, e=0.995$. 
Figure 2 gives $\left(A_{c} / B_{c}\right)^{1 / 2}$ vs $\varpi_{c}$ for the models existing in the literature (Einasto, 1970; Innanen, 1971). The quasi-invariance of $\left(A_{c} / B_{c}\right)^{1 / 2}$ on a large range of $\varpi_{c}$ is explained well by the discussion above, which also allows to interpret the differences between the curves for $\varpi_{c}<10 \mathrm{kpc}$ as connected with differences in the eccentricity of the spheroid and in the mass of the nucleus. (From this point of view, Einasto's and Schmidt's models with a nucleus and a spheroid, the surface boundary of which passes through the Sun, are directly comparable in this range of $\varpi_{c}$ : Einasto's spheroid is slightly less flattened and the nucleus mass is more important).

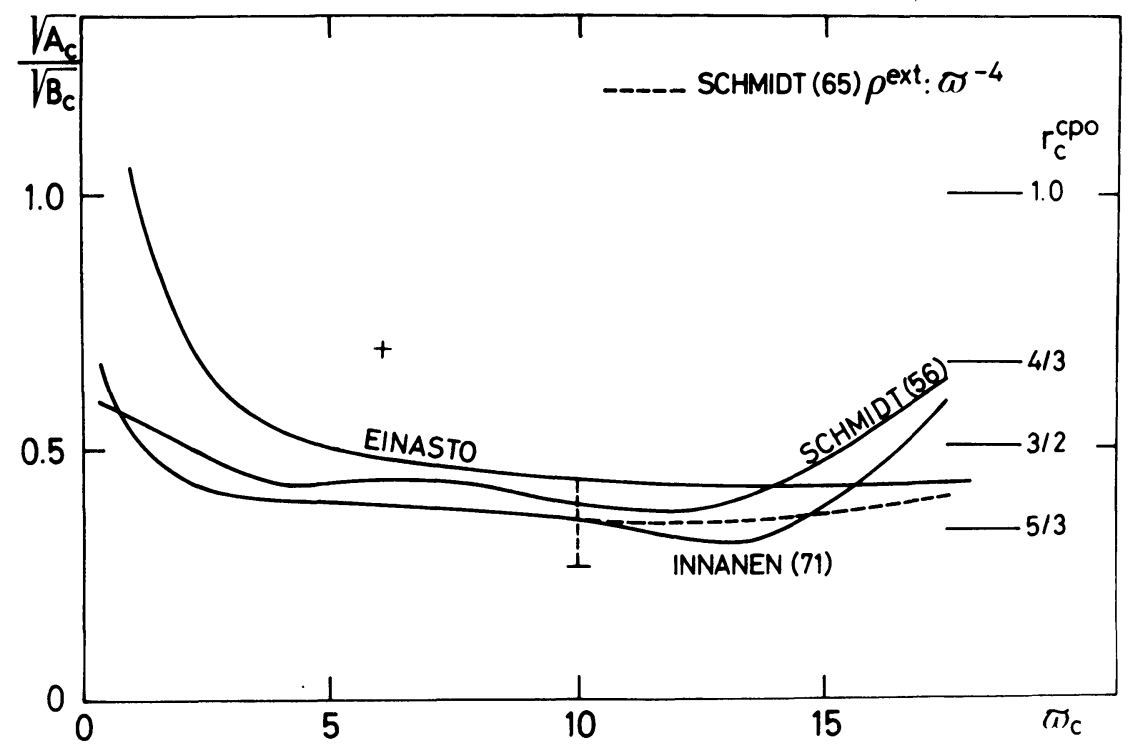

Fig. 2. $\left(A_{c} / B_{c}\right)^{1 / 2}$ vs $\varpi_{c}$ for different usual models. For the different symbols, see the text.

The vertical bar at $\varpi_{\mathrm{c}}=10 \mathrm{kpc}$ shows the possible values of $\left(A_{\mathrm{c}} / B_{\mathrm{c}}\right)^{1 / 2}$ which we have, taking into account the 'observational' uncertainty to be considered for $\varrho_{\odot}$ and with the standard values of Oort Constants $(A=15, B=-10)$. The upper limit corresponds to $\varrho_{\odot}=0.09 \mathscr{M}_{\odot} \mathrm{pc}^{-3}$ and the lower limit to $0.27 \mathscr{M}_{\odot} \mathrm{pc}^{-3}$. As the invariance of the ratio $\left(A_{c} / B_{c}\right)^{1 / 2}$ on a more or less large range of $\varpi_{c}$ is a general property of the models based on spheroids, we are able to guess what are the 'plateau' values of $\left(A_{c} / B_{c}\right)^{1 / 2}$ which we obtain, taking account of the errors on $\varrho_{\odot}$. We added a point $(\times)$ representing the value of $\left(A_{c} / B_{c}\right)^{1 / 2}$ which we obtain using the Oort constants recommended by Clube with $B_{\mathrm{c}}$ computed from $\varrho_{\odot}=0.15 \mathscr{M}_{\odot} \mathrm{pc}^{-3}$.

We infer from the diagram that no exact important resonance (corresponding to $\left(A_{c} / B_{c}\right)^{1 / 2}=\frac{2}{3}, \frac{1}{1}$ or $\left.\frac{4}{3}\right)$ is obtained for $\varpi_{c}$ not too small, unless rather extreme (but not excluded!) local galactic structure parameters are chosen.

\section{Resonant Orbits}

In non-linear problems such as the present one, the properties of the orbits do not 
depend critically on the exact value of $\left(A_{c} / B_{c}\right)^{1 / 2}$. If $\left(A_{c} / B_{c}\right)^{1 / 2}$ is 'near' a rational number $n / m$, we expect to find resonant periodic orbits closing after $n$ oscillations along the $w$-axis and $m$ oscillations along the $z$-axis. If the energy $E$ increases, $\left(A_{c} / B_{c}\right)^{1 / 2}$ is 'approached' by more and more rational numbers in the sense that $\mid\left(A_{c} / B_{c}\right)^{1 / 2}-$ $-(n / m) \mid$ is of the order of $E-E_{c}$, where $E_{c}$ corresponds to the circular energy. Hence, new types of resonant periodic orbits must appear. Our numerical explorations confirm this expectation. In both Schmidt potentials, orbits with $n / m=\frac{1}{1}$ or $\frac{4}{3}$ (not very near $\left.\left(A_{c} / B_{c}\right)^{1 / 2} \approx 0.4\right)$ end up in appearing provided the energy is high enough. Here we must emphasize that these rational values are the most important because they correspond to the periodic orbits which are surrounded by very important tubes in the configuration space. In a surface of section, more precisely in the phase plane $(\varpi, \dot{\varpi})$, these tubes give the most important islands, which may occupy a large part of the accessible region (see i.e. Mayer and Martinet, 1973, Figure 9). It would be extremely useful to establish the dependence of the range $\Delta$ of values of $n / m$ which produces resonant orbits with respect to the parameters characterizing the model. Unfortunately a theory giving $\Delta$ is available only for small values of $\varepsilon$ in the artificial 'galactic potential' $V=\frac{1}{2} A x^{2}+\frac{1}{2} B y^{2}-\varepsilon x y^{2}-\eta x^{3}$ (Contopoulos, 1973), where moreover $\eta$ is neglected. Thus the theoretical results are not applicable to our realistic galactic models, for which we often have rather large $\varepsilon$ 's and where $\eta$ is not always negligeable with regard to $\varepsilon$. However theory may indicate some qualitative tendency of behaviour for some functions of interest in our problem. For example, from the treatment of the orbits by the method of surface of section, we can use the notion of 'rotation number' (Contopoulos, 1970), that means the asymptotic value of the angle between two successive points of intersection $(\varpi, \dot{\varpi})$ of the trajectory with the plane $z=0$ in the phase plane, as seen from the 'central' invariant point $\varpi=\varpi_{c}, \dot{\varpi}=0$, which corresponds to the symmetric periodic orbit crossing the $\varpi$-axis always at the same point $\left(\varpi=\varpi_{c}, z=0\right)$. For this 'central' periodic orbit, we define the rotation number $r_{c p 0}$ as the limit of the rotation number of nearly non-periodic orbits. Further we also use the so-called characteristic diagram where symmetric periodic orbits are represented by a point $(E, \varpi)$, where $\varpi$ is the intersection point of the given orbit in the galactic plane $(z=0)$, with $\dot{\varpi}=0$ (see Paper II). Each family of such orbits.is identified by its rotation number $r=2-(n / m)$ if the orbit closes after $n$ radial oscillations and $m$ perpendicular oscillations. For $E$ increasing from $E=E_{c}$ as we move along the characteristic of the central family towards the intersection with characteristics of other families of order $m$, we tend for $r_{c p 0}$ to rational values $n^{\prime} / m=2-(n / m)$. The variation of $r_{c p 0}$ with $E$ gives the rate to which different resonant periodic orbits appear. Thus the slope $\mathrm{d} r_{c p 0} / \mathrm{d} E$ contains important information. Theory allows us to deduce an analytical form of it submitted to the condition that $\varepsilon$ is small but much larger than $\eta$. In fact from the formula given by Contopoulos (1968) for $r_{c p 0}$, we immediately deduce that

$$
\frac{\mathrm{d} r_{c p 0}}{\mathrm{~d} E}=\frac{\sqrt{A_{c}}}{\sqrt{B_{c}}} \frac{3 A_{c}-4 B_{c}}{2 A_{c} B_{c}^{2}\left(A_{c}-4 B_{c}\right)} \varepsilon^{2}
$$


which is of the order of $\varepsilon^{2} / B_{c}^{3}$ if $\left(A_{c} / B_{c}\right)^{1 / 2}$ is a constant. It can be shown (Contopoulos, 1970 ) that the range of values of $n / m$ for which resonant orbits appear is proportional to this quantity. This remark suggests to examine if the existence of a linear relation between $\Delta$, defined as $r_{c p 0}^{c}-r_{c p 0}^{\min }$ (where $r_{c p 0}^{c}=r_{c p 0}\left(E=E_{c}\right)$ and $r_{c p 0}^{\min }=$ minimum accessible value of $\left.r_{c p 0}\right)$ and $r^{\prime}=\mathrm{d} r_{c p 0} / \mathrm{d} E$ is a general property of more realistic galactic models, at least in the range of $\varpi_{c}$ where $\left(A_{c} / B_{c}\right)^{1 / 2}$ is approximately a constant.

The results of our numerical exploration for both Schmidt potentials show that this property is actually satisfied although the conditions of application of the theory are not filled in general. We emphasize that in both models the slope of the linear relation $\left(r^{\prime}, \Delta\right)$ is nearly the same. Starting from the idea that $r^{\prime}$ could give information for determining $\Delta$ in any model given we can examine what is the tendency of the sensitivity of this function to a changing of the model parameters, at least qualitatively.

Thus we express $r$ ' as a function of $C, v_{i}(i=-1$ and 1$), J_{n}$ for the 'spheroid + nucleus' model chosen previously, using (2), (4), (5) and (7). Detailed results are published in Paper III.

By considering values of $v_{i}$ which lead to reasonable shapes of the rotation curve $\Theta_{c}(\varpi)$, we may conclude (everything else being equal) that: (a) an increase of the mass of the nucleus leads to smaller values of $r^{\prime}$ for small $\varpi_{c} ;(b)$ if we take for instance a larger value of $v_{1}$ (with respect to Schmidt (1965) model), we have gradually larger values of $\Theta_{c}(\varpi)$ from $\varpi_{c}=3 \mathrm{kpc}$, which lead to a less sharp slope $r^{\prime}$ for $\varpi_{c}$ increasing; (c) if we take for $\varrho_{\odot}$ the value $0.09 \mathscr{M}_{\odot} \mathrm{pc}^{-3}$ instead of $0.15 \mathscr{M}_{\odot} \mathrm{pc}^{-3}, r^{\prime}\left(\varpi_{c}\right)$ becomes systematically slightly sharper. In order to be able to predict what values of $\Delta$ we can expect in a model for any given value of parameters, it would be necessary to have several numerical experiments which overlap the conditions of interest, to construct families of relations $\left(r^{\prime}, \Delta\right)$ parameterized either by $v_{-1}$ or by $v_{1}$ for instance.

A large value of $r^{\prime}$ could be a condition among others for the appearance of regions of instability in the phase plane $(\varpi, \dot{\varpi})$ ('wild' orbital behaviour). Another factor, important in this connection, may be guessed by considering the shape of the limit curve in the so-called characteristic diagram $(E, \varpi)$. The equation of this curve is

$$
E=\frac{1}{2} A_{c}\left(\varpi-\varpi_{c}\right)^{2}-\eta\left(\varpi-\varpi_{c}\right)^{3}+\cdots .
$$

Neglecting the terms of higher order, we observe that the ratio of both terms of the 2nd member is in the order of $\eta / A_{c}^{3 / 2}$. If $\eta / A_{c}^{3 / 2}$ is small, the curve becomes more concave and this fact can also contribute to congest the characteristics and lead to interactions of resonances and wild orbital behaviour. Of course this effect is enhanced if $r^{\prime}$ is larger. Further in the present context this conjecture is available for values of the energy not too far from $E=E_{c}$. However, here again, we can deduce a qualitative tendency of the sensitivity of $\eta / A_{c}^{3 / 2}$ to the parameters of our 'spheroid + nucleus' model. As for $\left(A_{c} / B_{c}\right)^{1 / 2}$ or $r^{\prime}$, we write $\eta / A_{c}^{3 / 2}$ for different $\varpi=\varpi_{c}$ as a function of the parameters $C, v_{i}, J_{n}$. Detailed results are published in Paper III. For the same set of values of these parameters as chosen before, we conclude:

(a) An increase of the mass of the nucleus leads to smaller values of $\eta / A_{c}^{3 / 2}$ for small $\varpi_{c}$ and could promote the appearance of interactions of resonances. 
(b) Gradually larger values of $\Theta_{c}(\varpi)$ from $3 \mathrm{kpc}$ (with respect to Schmidt (1965) values) leads to smaller values of $\eta / A_{c}^{3 / 2}$ for $\varpi_{c}>6 \mathrm{kpc}$ and could promote the appearance of interactions of resonances in these regions.

(c) $\eta / A_{c}^{3 / 2}$ is independent of the eccentricity of the spheroid and consequently of the value chosen for $\varrho_{\odot}$.

\section{Numerical Exploration in Both Schmidt Potentials and Conclusions}

From our systematic exploration of orbits in both Schmidt models for a grid of values of $J$ and $E\left(1.5<J<250\right.$ [units $10 \mathrm{~km} \mathrm{~s}^{-1} \mathrm{kpc}$ ], $E_{c}<E<-100$ [units $100 \mathrm{~km}^{2} \mathrm{~s}^{-2}$ ]) we emphasize the following points:

(1) For small $J$ 's we found always the main periodic orbits of order $m$, corresponding to the rotation number $r=2-(n / m)=\frac{4}{3}, \frac{1}{1}$ or $\frac{2}{3}$. As mentioned before, these values are related to the main islands in the phase plane $(\varpi, \dot{\varpi})$, which correspond to important tube orbits in the configuration space $(\varpi, z)$. For large $J(>75)$ we found the main periodic orbits corresponding to $r=\frac{3}{2}, \frac{4}{3}, \frac{1}{1}$. In Paper III we have presented complete statistics of families of periodic and tube orbits in the models we have considered.

(2) We found some interactions of resonances revealed by the dissolution of invariant curves in the phase plane, which correspond to a rather wild (or semi-ergodic) orbital behaviour in the configuration space, particularly for small $J$ 's. Table I summarizes the ranges of $r$ where such interactions are observed in each model. In fact, we ought to write ' $\cdots<r<\max \{r\}$ ', where $\max \{r\}$ is the maximum value of $r$, which always appears of the order of $r_{c p 0}^{c}$, obtained from Figure 2.

TABLE I

\begin{tabular}{rll}
\hline$J$ & Schmidt I & Schmidt II \\
\hline 1.5 & $\frac{1}{2}<r<r_{c p 0}^{c}$ & $\frac{1}{2}<r<r_{c p 0}^{c}$ \\
45 & $\frac{2}{3}<r<r_{c p 0}^{c}$ & $1<r<r_{c p 0}^{c}$ \\
105 & $\sim 0.9<r<r_{c p 0}^{c}$ & $\begin{array}{l}\text { Possible interactions } \\
\text { of high order }\left(r \sim r_{c p 0}^{c}\right)\end{array}$ \\
180 & $1<r<r_{c p 0}^{c}$ & No interaction detected \\
\hline
\end{tabular}

These phenomena occur at different values of $E$ (see for instance Paper I). We note that the range of interactions is generally more important in the first model. It is difficult to try to explain such differences in terms of our discussion in the previous section because of the structural differences between the models. On the contrary, we have verified the prediction concerning the influence of a more massive nucleus on quantities such as $r^{\prime}$ or $\eta / A_{c}^{3 / 2}$ : If we double the mass of the nucleus in the 2nd Schmidt model, we observe that for cases where $J$ is rather small (i.e. 20) the values of $r^{\prime}$ and $\eta / A_{c}^{3 / 2}$ graphically and numerically determined at $\varpi_{c}$ given, become weaker, especially 
for $\eta / A_{c}^{3 / 2}$. These modifications are accompanied by a clearer tendency to the dissolution of invariant curves for values of $E$ relatively not too far from $E_{c}$.

(3) The previous results, particularly those from Table I, reveal that anyone wanting to apply such considerations to some real galactic populations is faced by an apparently complicated situation. For instance, if we consider initial conditions corresponding to old disc or intermediate populations in the solar neighbourhood, characterized among others by $\bar{J}>\sim 150$, no 'wildness' probably appears in the orbital behaviour, whatever the mass model may be, even if the $\varpi$-velocity, $\dot{\varpi}$, is of the order of $150 \mathrm{~km} \mathrm{~s}^{-1}$. But for local halo objects $(\bar{J}<150)$, if we consider at $J$ given (say $J=45$ ) the values of the energy for which the dissolution begins, it is possible to find that the upper limit of $\dot{\varpi}$ which corresponds to good invariant curves in the phase plane (and consequently to the existence of a 'third' integral) is $150 \mathrm{~km} \mathrm{~s}^{-1}$ in Schmidt I but $250 \mathrm{~km} \mathrm{~s}^{-1}$ in Schmidt II! We must add that halo objects form a small fraction of the nearby star population and moreover few of them satisfy the conditions of motion which lead to a semi-ergodic behaviour. We could also emphasize that the observational fact suggesting the existence of a third isolating integral, that means the observed inequality of the meridional velocity dispersions, is also evident for the more metal-deficient local halo populations as RR Lyrae with $\Delta S>5$ or extreme subdwarfs, which have the highest velocities with respect to the Sun. Thus from a practical point of view we can consider that a semi-ergodic behaviour in the meridian plane is quite exceptional for observed objects in the solar neighbourhood. The situation is certainly more intricate for intermediate or halo populations in more central regions of the Galaxy where they are well represented. Unfortunately a confrontation of our results with real motions is impossible in such cases because kinematical data completely fail.

Finally we can think of some extensions of the first approach presented here concerning the sensitivity of the orbital behaviour to the model parameters:

(1) With the help of several numerical experiments a complete theory turning on a system of superposed heterogeneous spheroids, considered by some people as the best way of representing the Galaxy, could allow to specify the effects here conjectured.

(2) An 'up-to-date' massive halo could be included.

(3) Later, the same type of question (resonances, dissolution, etc.) could be asked with regard to a system with three degrees of freedom (a non axisymmetric galaxy with a potential $\Phi(\varpi, \vartheta, z)$. Such a study could concern old disc and perhaps intermediate galactic populations.

\section{References}

Contopoulos, G. and Hadjidemetriou, J. : 1968, Astron. J. 73, 86.

Contopoulos, G.: 1970, Astron. J. 75, 96.

Contopoulos, G.: 1973, in L. Martinet and M. Mayor (eds.), Dynamical Structure and Evolution of Stellar Systems, Third Advanced Course of the Swiss Society of Astronomy, Geneva Observatory, p. 52.

Einasto, J.: 1970, Tartu Astron. Obs. Teated, No. 26.

Innanen, K.: 1971, private communication.

Innanen, K. and Ryman, A. G.: 1972, Astrophys. Space Sci. 17, 447. 
Martinet, L. and Hayli, A.: 1971, Astron. Astrophys. 14, 103 (Paper I).

Martinet, L. and Mayer, F.: 1975, in preparation.

Mayer, F. and Martinet, L.: 1973, Astron. Astrophys. 27, 199 (Paper II).

Schmidt, M.: 1956, Bull. Astron. Inst. Neth. 13, 15.

Schmidt, M.: 1965, Stars and Stellar Systems 5, 513.

\section{DISCUSSION}

Contopoulos: What are the most important resonances, in the sense that the tube orbits occupy a large part of phase space?

Martinet: The main values of the rotation number $r=2-(r / m)$ which correspond to the most important islands in the phase plane are $\frac{1}{1}$ and $\frac{2}{3}$. It may happen that these islands occupy a very large part of the accessible region in this phase plane particularly for relatively small values of the angular momentum $J\left(\leqslant 1500 \mathrm{~km} \mathrm{~s}^{-1} \mathrm{kpc}\right)$.

Contopoulos: How do you think that these tube orbits might be observed?

Martinet: Tube orbits could be connected to the existence of stellar groups or drifts of stars as such as we observe in the solar neighbourhood for example. The most important tubes we described above ought rather to be related to larger structures in more extended regions than the very local solar neighbourhood and concern stellar samples with non-negligeable $z$-motions in certain cases.

Freeman: People have suggested that the inner parts of galactic disks could be hot, to stabilize the disks against barlike modes. Is this consistent with what you know about the third integral in realistic galactic potentials, e.g. take a star whose orbit has mean $\varpi \simeq 3 \mathrm{kpc}$, mean $\Pi \simeq 200 \mathrm{~km} \mathrm{~s}^{-1}$, mean $Z \simeq 50$ or $100 \mathrm{~km} \mathrm{~s}^{-1}$. Does this star have a third integral which keeps it in the disk (i.e. in $|Z| \leqslant 800 \mathrm{pc}$ ), or does it in fact orbit into a spheroidal volume, about the galactic centre?

Martinet: From our numerical exploration in both Schmidt potentials we can say that, for initial conditions in the range which you propound, we have some evidence that a third integral effectively keeps the motion in the disk, even if the $z$-velocity is $\sim 100 \mathrm{~km} \mathrm{~s}^{-1}$. The half thickness of the boxes occupied by the orbits is of the order of 500 to $800 \mathrm{pc}$, at most $1 \mathrm{kpc}$. These results seem to be independent of the potential chosen. 